\title{
The Relationship between Lateral Nasal Wall Collapse and Nasal Obstruction, a case-control study
}

\author{
Yong Ju Jang ${ }^{1}$, Mi Rye $\mathrm{Bae}^{2}$, and Woo Ri $\mathrm{Choi}^{3}$ \\ ${ }^{1}$ Asan Medical Center \\ ${ }^{2}$ Daejin Medical Foundation Bundang Jesaeng Hospital \\ ${ }^{3}$ Asan Medical Center, University of Ulsan College of Medicine
}

September 25, 2021

\begin{abstract}
Objectives In clinical practice, lateral nasal wall collapse during forced inspiration is widely regarded as a sign of nasal obstruction or criterion indicating nasal valve surgery. This study aims to evaluate the relationship between the degree of lateral nasal wall collapse and subjective nasal obstruction. Design Case-Control study Setting Tertiary centre hospital Participants Case group consisted of 24 patients who had been diagnosed with a deviated nasal septum or nasal valve stenosis. Control group consisted of 27 volunteers with no nasal obstruction symptoms and no septal deviation on nasal endoscopy. Main outcome measures Lateral nasal wall collapse is determined by the degree of lateral nasal wall triangle (LNWT) area reduction on frontal view during forced inspiration compared to quiet inspiration. LNWT area ratio of the patient and control groups was compared. The relationship between the lateral nasal wall collapse and clinical factors including symptom scores, nasal valve angles, skin thickness were evaluated. Results The average LNWT area ratio of the patient $(n=24)$ and control groups $(n=27)$ was 0.96 and 0.83 respectively $(\mathrm{p}=0.001)$. Symptom score (NOSE and VAS) is not related to the degree of lateral nasal wall collapse. Moreover, nasal valve angle and skin thickness were also not related to the degree of lateral nasal wall collapse. In 14 of the 19 patients, the more obstructed side corresponded to the side of narrower nasal valve angle, and 5 were not. Conclusion Lateral nasal wall collapse is not related to a patients' nasal obstruction.
\end{abstract}

\section{ABSTRACT}

Objectives

In clinical practice, lateral nasal wall collapse during forced inspiration is widely regarded as a sign of nasal obstruction or criterion indicating nasal valve surgery. This study aims to evaluate the relationship between the degree of lateral nasal wall collapse and subjective nasal obstruction.

Design

Case-Control study

Setting

Tertiary centre hospital

Participants

Case group consisted of 24 patients who had been diagnosed with a deviated nasal septum or nasal valve stenosis. Control group consisted of 27 volunteers with no nasal obstruction symptoms and no septal deviation on nasal endoscopy.

Main outcome measures 
Lateral nasal wall collapse is determined by the degree of lateral nasal wall triangle (LNWT) area reduction on frontal view during forced inspiration compared to quiet inspiration. LNWT area ratio of the patient and control groups was compared. The relationship between the lateral nasal wall collapse and clinical factors including symptom scores, nasal valve angles, skin thickness were evaluated.

Results

The average LNWT area ratio of the patient $(n=24)$ and control groups $(n=27)$ was 0.96 and 0.83 respectively $(\mathrm{p}=0.001)$. Symptom score (NOSE and VAS) is not related to the degree of lateral nasal wall collapse. Moreover, nasal valve angle and skin thickness were also not related to the degree of lateral nasal wall collapse. In 14 of the 19 patients, the more obstructed side corresponded to the side of narrower nasal valve angle, and 5 were not.

Conclusion

Lateral nasal wall collapse is not related to a patients' nasal obstruction.

\section{Five key points}

Control group exhibited a larger degree of lateral nasal wall collapse compared with the case group, the LNWT average area ratio of the case and control groups was 0.96 and 0.83 , respectively.

Nasal obstruction symptom scores (VAS and NOSE) were not related to the degree of lateral nasal wall collapse.

Nasal valve angle was not related to the degree of lateral nasal wall collapse.

Nasal skin thickness was not significantly related to the degree of lateral nasal wall collapse.

The associated factor for nasal obstruction in this study was the sides with narrower nasal valve angle.

\section{Keywords}

Nasal obstruction, Lateral nasal wall collapse, Nasal valve, Forced inspiration

\section{Introduction}

Nasal obstruction is one of the most common symptoms managed in otolaryngology practice. The nasal valve is the narrowest segment in the nasal cavity. Anatomically, the nasal valve area consists of the caudal border of the upper lateral cartilage, nasal septum, nasal floor, and the end of the inferior turbinate(1). A narrow or closed nasal valve could cause nasal obstruction(2). Many tools have been used to evaluate nasal valve function, including rhinoscopy, nasal endoscopy, modified Cottle's maneuver, acoustic rhinometry, and computed tomography. However, no gold standard test is being used to accurately and objectively diagnose nasal valve dysfunction(3).

In clinical practice, many patients having a stuffy nose demonstrate the collapse of the lateral nasal wall and try to express how much they are suffering from nasal obstruction. Even surgeons tend to regard nasal wall collapse during forced inspiration as an indication criterion of nasal valve surgery(4). However, the relationship between the lateral nasal wall collapse and nasal obstruction in patients is not well understood.

This study aims to evaluate the relationship between the degree of lateral nasal wall collapse and subjective nasal obstruction. In addition, the clinical significance and diagnostic value of the lateral nasal wall collapse were investigated.

\section{Materials and Methods}

\section{Patients}

The medical records of the patients who visited the otolaryngology clinic at Blinded for review with complaints of nasal obstruction from December 2019 to June 2020 were reviewed. Patients who had been diagnosed with a deviated nasal septum or nasal valve stenosis and had undergone subsequent surgery aimed 
to correct nasal breathing function were included in the case group. The surgery performed on the patients included septoplasty, open rhinoseptoplasty, and valve surgery. Patients who had a severe skin disease, nostril stenosis, sinusitis, and nasal polyposis were excluded. Consequently, 24 patients met the inclusion criteria. The control group consisted of 27 volunteers with no nasal obstruction symptoms and no septal deviation on nasal endoscopy. All patients in the case group had an ostiomeatal unit computed tomography (OMU CT) scan before surgery, and no computed tomography (CT) scan was taken in the control group. Moreover, demographic data, symptom score questionnaires, surgical records, and facial videos were collected from the participants.

This study was approved by the Institutional Review Board ofBlinded for review , and all participants agreed to take facial imaging and signed informed consent. (IRB No. 2020-0160).

\section{Measuring nasal valve angle and skin thickness}

The OMU CT scans were obtained using a 128-slice CT machine (SOMATOM Definition AS, Siemens Healthcare, Erlangen, Germany) at a 2-mm slice thickness. CT scans showed the nasal valve angle degree. The nasal valve angle was anatomically determined between the nasal septum and caudal border of the upper lateral cartilage. The angle of the nasal valve on each side was measured on the coronal plane of the CT image. Imaging of the level of the nasal valve showed the anterior end of the inferior turbinate (Figure 1.A). Nasal skin thickness was measured on the axial plane of OMU CT scans. Bilateral nasal skin thickness at the rhinion and nostrils were measured by an in-house software (PetaVision;Blinded for review Figure 1. B, C).

\section{Subjective nasal obstruction}

Nasal Obstruction Symptom Evaluation (NOSE) and visual analog scale (VAS) questionnaires were collected from all participants. The NOSE score consists of five questions concerning subjective assessment of the nasal obstruction. Each question can be answered on a 5-point scale from 0 (not a problem) to 4 (severe problems). The patient's score can be scaled from 0 to 100 after multiplying the raw score by 5 . For the VAS score, the participant was asked to choose the scale (1-10) that best fits the nasal obstruction severity.

\section{Video}

Facial videos were obtained from all participants with informed consent. This video was taken with iPhone 7's rear camera $30 \mathrm{~cm}$ in front of the face. The Frankfort line (the line connecting the tragal cartilage with the upper margin of the external auditory canal) was placed horizontally in the center of the video. Quiet and forced inspiration videos were simultaneously recorded. All participants were asked to breathe quietly as usual at first and breathe intensely the second time. The degree of lateral wall collapse during inspiration was measured using the video image by the method described below.

\section{Measurement of lateral nasal wall collapse}

Images in this video were captured during moments of quiet and forced inspiration. Forced inspiration photos were captured when the lateral nasal wall was depressed the most. A simple method was devised to calculate the degree of the lateral nasal wall collapse. First, the tip-defining point (TDP) in the captured image was marked. Lines were then vertically and horizontally drawn on the TDP. The point where the vertical line meets the interpupillary line was called point a. A hypotenuse was made by connecting the point where the alar crease ended on the same side in a. The point where the horizontal line and hypotenuse met becomes point $b$. In this study, a right-angled triangle with three vertices of a, b, and TDP was called the lateral nasal wall triangle (LNWT; Figure 2). The length of the three sides of a triangle was measured using the software Picpick v5.1.3 (NGWIN). The area-to-area ratio was calculated as follows:

\footnotetext{
LWNT area $=$ base $\times$ height $\times \frac{1}{2}$

Area ratio $=\frac{\text { area in forced inspiration }}{\text { area in quiet inspiration }}$

Statistical analysis
} 
All statistical analyzes were performed using the Statistical Package for the Social Sciences (SPSS), version 21 (IBM, Chicago, IL, USA). Statistical significance was defined as $P$ value $<0.05$. The difference in lateral wall collapse between the case and control groups was compared using the $t$-test. The gamma-kappa test was used to assess the agreement in subjective symptoms, lateral nasal wall collapse, and nasal valve. Moreover, the relationship between the symptom score, lateral nasal wall collapse, and nasal valve angle was analyzed through a correlation test.

\section{Results}

Patient demographics for each group are shown in Table I. No statistically significant differences in age and gender between the two groups were noted. In the case group, 3, 1, and 20 patients had right-side, left-side, and bilateral nasal obstructions, respectively. Of the 20 patients, the right-side nostril felt more clogged than the left in 3 patients, 12 felt more in the left side, and the other 5 felt the same degree of obstruction in both sides. The subjective nasal symptom score is also shown in Table I. NOSE and VAS scores were significantly higher in the case than in the control group.

Lateral nasal wall collapse: Case vs. control group

The degree of lateral nasal wall collapse can be determined by the degree of LNWT area reduction during forced inspiration compared to quiet inspiration. Moreover, the LNWT area ratio of the case and control groups was compared. This analysis included 48 and 54 areas from the case and control groups, respectively. The average area ratio of the case and control groups was 0.96 and 0.83 , respectively. The area of the case group decreased by $4 \%$ during forced inspiration compared to the quiet inspiration. However, this decrease was not statistically significant $(p=0.342)$. The area of the control group decreased by $17 \%$ compared to the quiet inspiration, which was statistically significant (Table II). Statistically significant difference was noted in the area ratio between the case and control groups $(p=0.001)$.

\section{Symptoms of nasal obstruction and lateral nasal wall collapse}

Of the 24 patients in the case group, 19 had a unilaterally dominant nasal obstruction and 5 reported that both noses were blocked to a similar extent. In these 19 patients, the ratio of the LNWT area on the more clogged side was compared with the less clogged side. The area ratio of the more and less clogged sides was 0.92 and 1.00, respectively, with no noted significant difference $(p=0.145)$, In 12 of the 19 patients, the more clogged side corresponded to the side of more lateral nasal wall collapse, and 7 were not. The degree of agreement between the more clogged side and the side in which more lateral nasal wall collapse occurred was analyzed with a gamma-kappa agreement test. The sides did not match $(\kappa=0.304, p=0.127)$. When the relationship between the symptom score (NOSE scale and VAS score) and LNWT area ratio was analyzed, neither is related $(p=0.303$ and $p=0.653)$.

Symptom of nasal obstruction and valve angle

The side with more obstruction of the nose was also compared to the side with the narrower nasal valve angle on the OMU CT scan. Of the 24 patients in the case group, 19 patients with unilateral dominant nasal obstruction were included in the analysis. In 14 of the 19 patients, the more obstructed side and narrower nasal valve angle were similar, and 5 were on the other side. Concordance was analyzed using the gamma-kappa test, and the kappa value was 0.463 , indicating moderate agreement (Table III). The relationship between the symptom scores and nasal valve angles on the narrower side was analyzed using Spearman correlation analysis. In 19 patients with a unilaterally dominant nasal obstruction in the case group, both NOSE and VAS scores had no statistically significant relationship with the nasal valve angles $(p=0.154$ and $p=0.352)$.

Lateral nasal wall collapse and valve angle

The relationship between nasal valve angle and lateral nasal wall collapse was evaluated. This analysis included 48 areas in 24 patients in the case group. The average nasal valve angle in the 48 areas was $11.01^{\circ}$. 
Spearman correlation analysis was used to evaluate the correlation between the angle of the nasal valve and area ratio of the collapse of the lateral nasal wall; it was also found to not correlate $(p=0.351)$. (Table IV)

Lateral nasal wall collapse and skin thickness

Nasal skin thickness at the rhinion and the nostrils were measured in the axial cut of the OMU CT. The relationship between skin thickness and lateral wall collapse was analyzed. However, the skin thickness was not significantly related to the degree of lateral nasal wall collapse $(p=0.972$ and $p=0.993$, at the rhinion and the nasal tip, respectively) (Table IV)

\section{Discussion}

This study confirmed that no significant correlation exists between nasal obstruction symptom scores and the degree of lateral nasal wall collapse. The lateral nasal wall collapsed more in the normal subject than in the patient group with nasal obstruction.

Physiologic lateral nasal wall collapse is hardly distinguished from pathologic collapse. In a study of dynamic changes in nasal valve movement using MR-Cine imaging technique, the authors reported that the nasal valve angle was narrowed on average to $68 \%$ of the baseline angle upon forced inspiration in normal subjects with no nasal obstruction symptoms(5). Chang suggested that lateral wall collapse itself should not be interpreted as nasal obstruction caused by nasal valve dysfunction because lateral nasal wall collapse during inspiration may be a physiological phenomenon that reflects the simple collapsibility of the nasal structure(6). However, despite the lack of sufficient evidence, both patients and doctors conventionally considered lateral nasal wall collapse as a sign of nasal obstruction or criterion indicating nasal valve surgery. In a survey of otolaryngologists, nearly half of the respondents identified visible valve collapse on inspiration, as the most important finding suggesting the need for nasal valve repair(4). This study is meaningful in that the results indicate that lateral nasal wall collapse may not indicate a patients' nasal obstruction.

The primary result of this study suggests that the control group exhibited a larger degree of lateral nasal wall collapse compared with the case group. Nasal wall collapse is determined by the interaction of the three main factors: atmospheric pressure outside the nostril, static pressure in the nasal cavity, and elasticity (rigidity) of the nasal wall(7). According to the Bernoulli equation(8), the static pressure tends to decrease in the nasal valve area, where the velocity increases due to the reduced cross-sectional area. The lateral wall may collapse inward in the presence of a large pressure drop occurring in the nasal valve area. Forced inspiration would not induce much larger air velocity or equivalently much lower pressure in the airway than quiet inspiration because the case group is characterized by persistent nasal obstruction. On the other hand, forced inspiration would allow much larger velocity or equivalently much lower pressure in the control group, which exhibits normal nasal patency. Therefore, the larger pressure difference (between atmospheric pressure and the pressure in the airway) developed in the control group in forced inspiration is likely to cause greater nasal wall collapse ${ }^{6}$.

Neither the sides of nasal obstruction nor symptom scores were related to lateral nasal wall collapse in the case group. The only factor associated with nasal obstruction in this study was the sides with narrower nasal valve angle. In 14 of 19 patients, the more clogged side of the nostrils coincided with the sides with a narrower nasal valve. However, no linear correlation between symptom score and nasal valve angle was noted. Attempts have been made to find the objective factors associated with nasal obstruction. Verhoeven et al. demonstrated that the type and severity of septal deviation were not related to the degree of subjective nasal obstruction(9). Shafik et al. reported that no correlation was noted between nasal valve angle and NOSE score measurement(3). Furthermore, Lee et al. reported a correlation between the degree of subjective nasal obstruction and anatomical features of the posterior nasal cavity(10). The perception of nasal patency is the result of several complex physical and sensory factors (e.g., mucosal heating, humidity, nasal resistance, and nasal trigeminal sensitivity $(8,11)$. Thus, finding objective anatomical factors that are directly related to nasal obstruction is difficult.

This study found that the nasal valve angle and skin thickness were also not related to lateral nasal wall 
collapse. A few studies exist on the collapsibility of the lateral nasal wall. Maalouf et al. reported that the difference between the absolute value of inspiratory and expiratory flow was significantly higher in the nasal valve collapse than in the no collapse group(12). Studies on the biomechanical evaluation of nasal skin have shown that the skin of the patients with nasal valve collapse appears to retain elasticity, but has increased flexibility compared with the nasal skin without valve collapse(13). However, further studies on lateral nasal wall collapse concerning skin thickness are needed.

This study has several limitations. First, whether the lateral nasal wall collapse caused by forced inspiration can represent the patient's real symptoms in daily life is questionable. Forced inspiration, except for strenuous exercise, is rare in everyday life. Moreover, the speed and amount of inhalation airflow varies in patients and may vary depending on the time and place. Second, the methodological problem is another limitation. A grading system for lateral nasal wall collapse based on an endoscopic intranasal view according to the medial movement of the lateral nasal wall was $\operatorname{proposed}(14,15)$. Moreover, Choi diagnosed lateral wall collapse when the patient had nostril obstruction at the basal view during forced inspiration(16). A new method for measuring the degree of collapse by comparing the quiet and forced video image of inspiration was devised because few studies have previously quantified the degree of lateral nasal wall collapse. Anatomical landmarks were drawn and measured based on manually captured video images, which may result in poor reproducibility.

Third, all participants in this study were Korean. It is likely that Caucasian patients with a tall narrower nose would have much more significant nasal airway obstruction upon forced inspiration(17). Asian patients have a wider nasal valve angles and thicker nasal sidewalls that are better able to resist collapse upon forced inspiration. Hence, the different nasal anatomy between the Asian and Caucasian may limit the generalization of our study results.

\section{Conclusions}

In conclusion, the lateral nasal wall collapsed more in normal subjects than in patients with nasal obstruction. The collapse of the lateral nasal wall was not related to the degree of obstruction symptoms, angle of the nasal valve, or nasal skin thickness. The only associated factor for nasal obstruction in this study was the sides with narrower nasal valve angle. Thus, the clinical significance of lateral nasal wall collapse needs to be reconsidered.

\section{References}

1. Bridger GP. Physiology of the Nasal Valve. Archives of Otolaryngology. 1970;92(6):543-53.

2. Yarlagadda BB, Dolan RW. Nasal valve dysfunction: diagnosis and treatment. Current Opinion in Otolaryngology \& Head and Neck Surgery. 2011;19(1):25-9.

3. Shafik AG, Alkady HA, Tawfik GM, Mohamed AM, Rabie TM, Huy NT. Computed tomography evaluation of internal nasal valve angle and area and its correlation with NOSE scale for symptomatic improvement in rhinoplasty. Brazilian Journal of Otorhinolaryngology. 2020;86:343-50.

4. Wang Y, Bonaparte JP. Diagnosis and management of septal deviation and nasal valve collapse - a survey of Canadian otolaryngologists. Journal of Otolaryngology - Head \& Neck Surgery. 2019;48(1):71.

5. San Nicoló M, Berghaus A, Jacobi C, Kisser U, Haack M, Flatz W. The nasal valve: new insights on the static and dynamic NV with MR-imaging. European Archives of Oto-Rhino-Laryngology. 2020;277(2):463-7.

6. Chang J. Surgery for Nasal Valve Compromise. Korean Journal of Otorhinolaryngology-Head and Neck Surgery. 2014;57(4):214-25.

7. Grützenmacher S, Günther M, Robinson DM, Mlynski G, Beule A. Investigations for the Diagnostic Recording of Nasal Wing Collapse. The Laryngoscope. 2005;115(10):1763-7.

8. Hsu DW, Suh JD. Anatomy and Physiology of Nasal Obstruction. Otolaryngologic Clinics of North America. 2018;51(5):853-65. 
9. Verhoeven S, Schmelzer B. Type and severity of septal deviation are not related with the degree of subjective nasal obstruction. Rhinology. 2016;54(4):355-60.

10. Lee DC, Shin JH, Kim SW, Kim SW, Kim BG, Kang JM, et al. Anatomical analysis of nasal obstruction: nasal cavity of patients complaining of stuffy nose. The Laryngoscope. 2013;123(6):1381-4.

11. Zhao K, Jiang J, Blacker K, Lyman B, Dalton P, Cowart BJ, et al. Regional peak mucosal cooling predicts the perception of nasal patency. The Laryngoscope. 2014;124(3):589-95.

12. Maalouf R, Bequignon E, Devars du Mayne M, Zerah-Lancner F, Isabey D, Coste A, et al. A functional tool to differentiate nasal valve collapse from other causes of nasal obstruction: the FRIED test. Journal of Applied Physiology. 2016;121(1):343-7.

13. Bonaparte JP, Campbell R. Assessment of Pliability and Elasticity of the External Nasal Skin in Patients With Unilateral Nasal Valve Collapse. JAMA Facial Plastic Surgery. 2018;20(6):475-9.

14. Most SP. Trends in functional rhinoplasty. Arch Facial Plast Surg. 2008;10(6):410-3.

15. Stolovitzky P, Sidle DM, Ow RA, Nachlas NE, Most SP. A prospective study for treatment of nasal valve collapse due to lateral wall insufficiency: Outcomes using a bioabsorbable implant. The Laryngoscope. 2018;128(11):2483-9.

16. Choi IJ, Kim KH, Kim NY, Lee MC. Diagnosis and Treatment of Dynamic External Nasal Valve Collapse: Application of Alar Batten Graft and Alar Flaring Suture. Korean J Otorhinolaryngol-Head Neck Surg. 2016;59(7):551-6.

17. Suh M-W, Jin H-R, Kim JH. Computed tomography versus nasal endoscopy for the measurement of the internal nasal valve angle in Asians. Acta Oto-Laryngologica. 2008;128(6):675-9.

\section{Hosted file}

Figure Legends_Jang, Bae.docx available at https://authorea.com/users/436570/articles/538795the-relationship-between-lateral-nasal-wall-collapse-and-nasal-obstruction-a-case-

control-study 


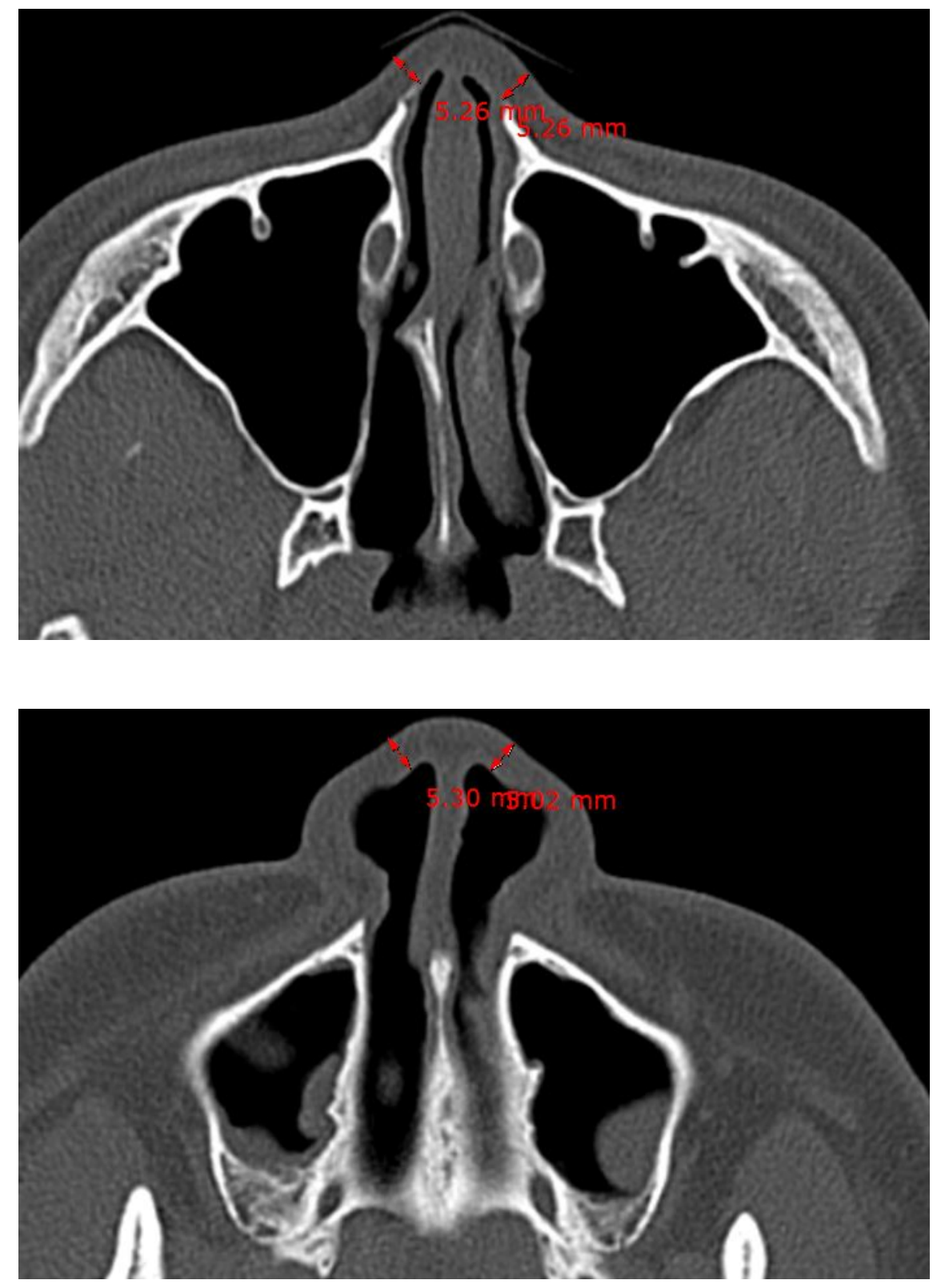


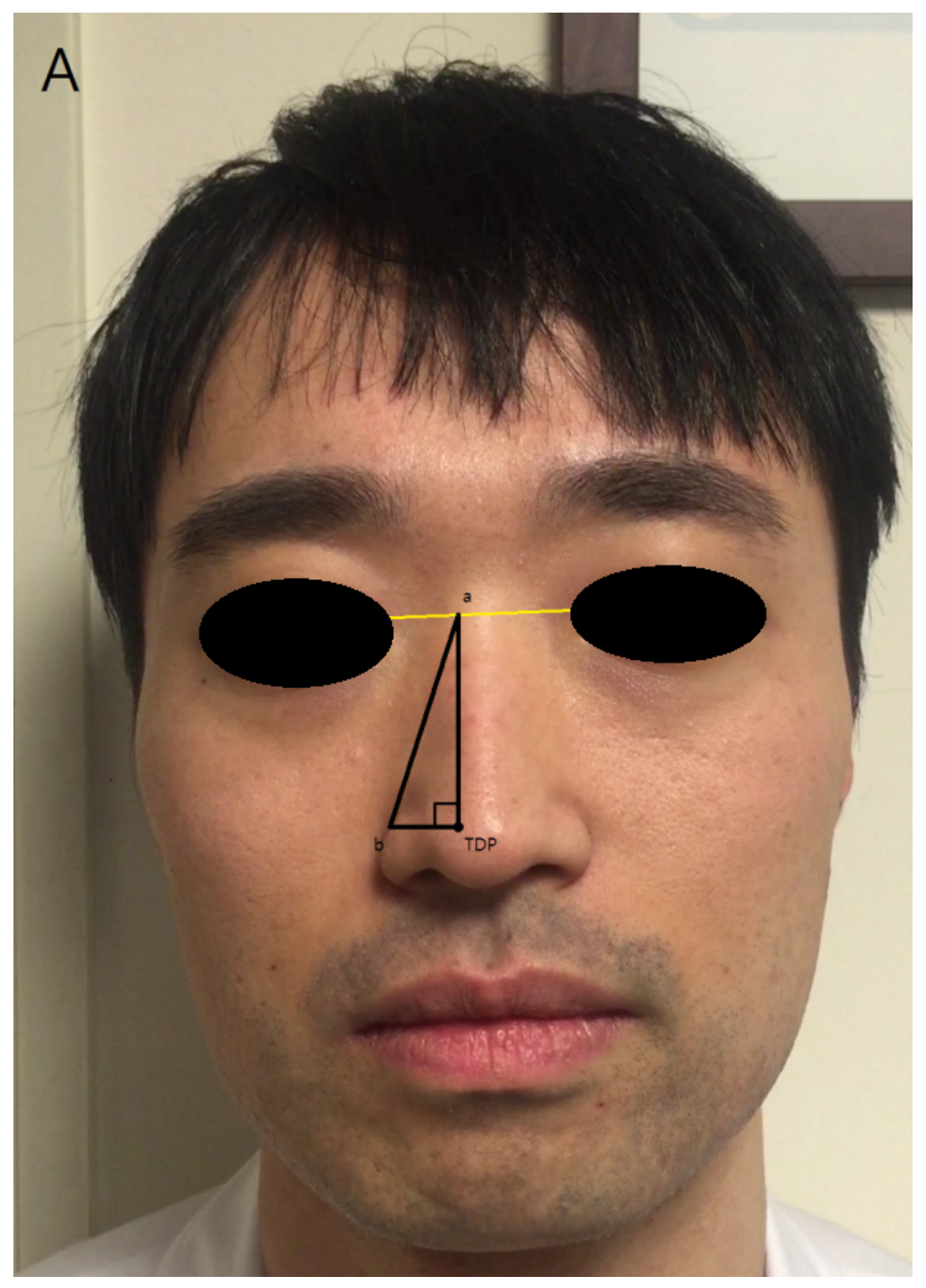




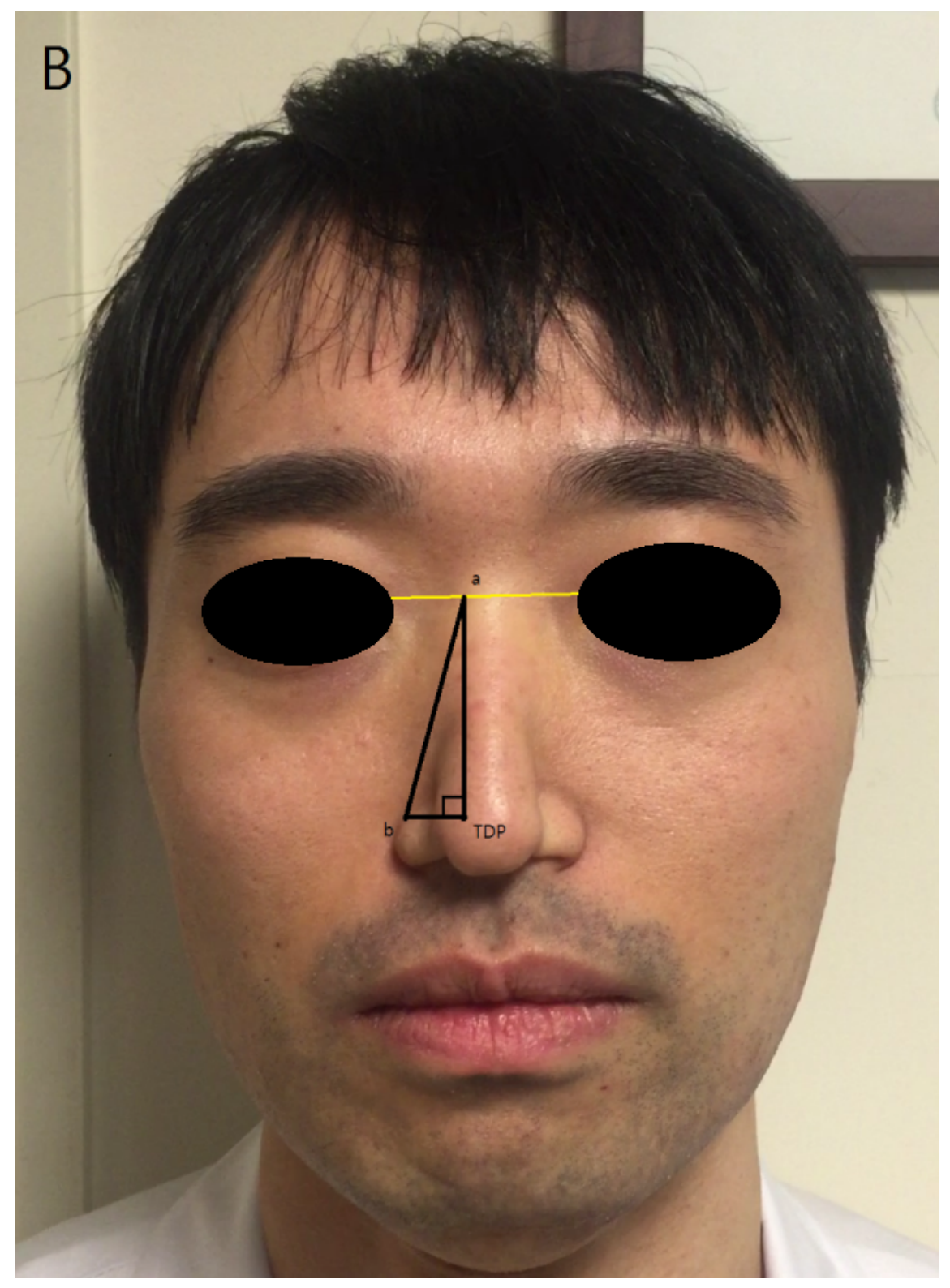

\section{Hosted file}

Tables 20210826.docx available at https://authorea.com/users/436570/articles/538795-therelationship-between-lateral-nasal-wall-collapse-and-nasal-obstruction-a-case-controlstudy 